Research Article

Eun-Jim Sim, Marcel Harpaintner, Markus Kiefer

\title{
Is subliminal face processing modulated by attentional task sets? Evidence from masked priming effects in a gender decision task
}

https://doi.org/10.1515/psych-2020-0006

Received June 5, 2019; accepted January 20, 2020

\begin{abstract}
Unlike classical theories of automaticity, refined theories suggest that unconscious automatic processes depend on cognitive control settings. Cognitive control influences on unconscious word and object processing are well documented, but corresponding findings in the field of face processing are heterogeneous. The present study therefore investigated, whether subliminal face priming in a gender categorization task is susceptible to feature-specific attention. Participants performed a gender decision task by orthogonally varying gender congruency (prime-target: same vs. different gender) and emotion congruency (prime-target: same vs. different emotional facial expression) using a masked priming paradigm. Perceptual vs. emotional induction tasks, performed prior to prime presentation, served to activate corresponding attentional task sets. Subliminal gender priming (faster reactions to gendercongruent primes) differed as a function of induction task and emotional congruency. Following perceptual induction, gender priming was only obtained in the emotionally congruent condition, whereas following emotional induction gender priming was observed independently of emotional congruency. In line with the classical notion of automaticity, subliminal gender priming did not depend on a specific attentional focus. However, attention to shape facilitated subliminal processing of task-irrelevant emotional facial expressions. Most likely, mutual facilitation of emotionally congruent prime and target representations enhanced gender priming compared with emotionally incongruent pairings.
\end{abstract}

Keywords: consciousness, automatic processes, attentional control, subliminal perception, subliminal priming of faces

\section{Introduction}

Classical theories of automaticity assume that unconscious automatic processes are triggered unintentionally and independently of higher level cognitive influences (Posner \& Snyder, 1975; Schneider \& Shiffrin, 1977) such as capacity-limited attentional resources or task demands. Cognitive control is therefore assumed to exclusively operate in the conscious domain. Refined theories of automaticity, in contrast, have challenged this classical association between consciousness and cognitive control (Kiefer \& Martens, 2010; Neumann, 1984; Spruyt, De Houwer, \& Hermans, 2009). Instead, this class of theories proposes that even unconscious automatic processing is susceptible to cognitive control influences to some extent. Modulatory cognitive control influences on unconscious automatic word and object processing have meanwhile been well documented (for reviews, see Ansorge, Kunde, \& Kiefer, 2014; Kiefer, 2012, 2018). However, corresponding

*Corresponding author: Eun-Jim Sim, Ulm University, Department of Psychiatry and Psychotherapy III, Leimgrubenweg 12, 89075 Ulm, Germany, E-mail: eun-jin.sim@uni-ulm.de

Marcel Harpaintner, Markus Kiefer, Ulm University, Department of Psychiatry and Psychotherapy III, Ulm, Germany

כ Open Access. ( 2020 Eun-Jim Sim et al., published by De Gruyter. $@$ Br tion 4.0 Public License. 
findings in the field of unconscious human face processing are heterogeneous (Finkbeiner \& Palermo, 2009; Khalid \& Ansorge, 2017; Khalid, Finkbeiner, Konig, \& Ansorge, 2013), but seem partially in line with the classical theoretical stance of automaticity. It is therefore discussed whether faces are partially processed along specific functional and neural pathways independent of cognitive control circuits, possibly due to the biological importance of this stimulus class (Ansorge, et al., 2014). To contribute to the debate about cognitive control influences on unconscious face processing, the current research investigated, whether cognitive control settings modulate unconscious automatic face processing.

An important method to study unconscious automatic processing is the subliminal priming paradigm, in which briefly presented, invisible masked primes trigger automatic processes that facilitate reactions to subsequent targets (Dell'Acqua \& Grainger, 1999; Draine \& Greenwald, 1998; Lamy, Mudrik, \& Deouell, 2008). With masked word and object stimuli as primes and targets, subliminal priming paradigms have revealed unconscious visuo-motor (Klotz \& Neumann, 1999; Vorberg, Mattler, Heinecke, Schmidt, \& Schwarzbach, 2003), semantic (Kiefer, 2002; Kiefer \& Spitzer, 2000; Marcel, 1983) and emotional processing (Duckworth, Bargh, Garcia, \& Chaiken, 2002; Öhman, Flykt, \& Esteves, 2001). Such subliminal priming effects arising from masked primes were not only observed for words and object pictures, but also for faces. For instance, in emotional expression (happy vs. fearful) or in gender categorization tasks (female vs. male face) masked priming effects were observed with faster responses, when prime target pairings were emotionally congruent (e.g., prime and target display a happy face) or gender congruent (e.g., prime and target show a female face) (Dimberg \& Petterson, 2000; Johnson, 2005; Kiss \& Eimer, 2008; Palermo \& Rhodes, 2007). This indicates that emotional expression and gender information of faces can be processed at an unconscious level.

There is evidence that unconscious face processing might involve a subcortical-cortical route based on the projection of the retina to the cortex via midbrain's superior colliculi bypassing primary visual cortex (de Gelder, Vroomen, Pourtois, \& Weiskrantz, 1999; Jolij \& Lamme, 2005; Khalid, et al., 2013). This subcortical route receives input mostly from the magnocellular pathway, which predominantly codes low spatial frequencies. In line with this assumption, only faces containing low spatial frequencies elicited subliminal priming effects in a gender decision task (Finkbeiner \& Palermo, 2009; Khalid, et al., 2013). Furthermore, these subliminal face priming effects based on low spatial frequencies did not depend on spatial attention (Finkbeiner \& Palermo, 2009; Khalid, et al., 2013). This suggests that the subcortical pathway in unconscious face processing does not require attentional amplification. However, in addition to subcortical processing, there are also cortical routes of unconscious processing (Pessoa \& Adolphs, 2010), which seem to depend on attentional amplification. Masked object priming effects in an animacy categorization task and gender priming effects of faces containing only high spatial frequency information, which both presumably depend on processing in cortical pathways, were only observed at attended locations (Finkbeiner \& Palermo, 2009; Khalid, et al., 2013).

This observation of attentional effects on subliminal object priming is in line with other research within the field of unconscious cognition indicating that even unconscious automatic processing depends on cognitive control settings, which sensitize relevant processing pathways (for reviews, see Ansorge, et al., 2014; Kiefer, 2012). Both the attentional sensitization model (Kiefer \& Martens, 2010) as well as the featurespecific attention allocation account (Spruyt, et al., 2009) propose that unconscious automatic processes, which are congruent with attentional task representations, are amplified, whereas other processes are attenuated. This notion of attentional modulation by task representations can be tested with the induction task paradigm (Kiefer \& Martens, 2010). In this paradigm, a masked subliminal priming task is preceded by an induction task to induce a specific task set. For instance, subliminal semantic priming effects of words within a lexical decision task, which depend on unconscious semantic prime processing, were only found following a semantic induction task (living/non-living classification), but not following a perceptual induction task (round/elongated classification) (Kiefer \& Martens, 2010; Martens, Ansorge, \& Kiefer 2011; Martens \& Kiefer, 2009). Furthermore, subliminal visuo-motor response priming of visual objects, which requires an arbitrary association of a visual shape with a response, was only obtained subsequent to a perceptual shape, but not subsequent to a semantic (Martens, et al., 2011) or a color induction task (Zovko \& Kiefer, 2013). This shows that unconscious priming requires a differential attentional focus depending on the priming-relevant stimulus dimension (e.g., perceptual shape vs. semantic meaning). 
The present study extended this previous line of research on attentional influences on unconscious cognition and investigated, whether subliminal face priming in a gender categorization task is susceptible to feature-specific attention. As described above, earlier studies on subliminal face processing manipulated spatial attention and found masked face priming in a gender categorization task, even when primes appeared at an unattended spatial location (Khalid, et al., 2013; Palermo \& Rhodes, 2007). This observation suggests that unconscious processing of facial stimuli, in contrast to objects or words, is not susceptible to attentional control mechanisms, possibly due to the involvement of the subcortical route (Ansorge, et al., 2014). However, the influence of feature-specific attention on subliminal face priming has not been investigated in previous work. The present study, therefore, used the induction task paradigm to manipulate the attentional focus prior to subliminal prime face presentation. Participants were presented with visible female and male faces as targets, which had to be categorized according to their gender. Target faces were preceded by invisible masked gender-congruent or -incongruent prime faces.

In order to test whether the task-irrelevant emotional expression dimension of a face is also unconsciously processed, we presented female and male faces with happy and fearful facial expressions as primes and targets by crossing the factors gender- und expression congruency. Although dimensions of a masked stimulus typically only elicit priming effects when they are task-relevant (Ansorge \& Neumann, 2005; Eckstein \& Perrig, 2007), it might be possible that the task-irrelevant emotional expression dimension interacts with gender congruency. Recently, Schmitz and Wentura (Schmitz \& Wentura 2012; Schmitz, Wentura, \& Brinkmann, 2014) suggested that emotionally congruent prime target pairings mutually facilitate each other, leading to increased prime activation maintenance. In line with this theoretical reasoning, they found larger effects of semantic congruency for evaluatively congruent than for evaluatively incongruent prime-target pairs. This influence of the task-irrelevant emotional expression dimension might be more pronounced, when attention is directed to emotional stimulus features.

Prior to the subliminal prime presentation, we therefore presented participants with two types of induction tasks, which should direct feature-specific attention either on the perceptual shape of a stimulus (perceptual induction task) or on the emotional content of the stimulus. As an emotional induction task, we used an arousal decision task (emotionally arousing vs. neutral stimulus). We administered an arousal decision task and not a valence decision task (positive vs. negative emotional meaning), in order to increase attention to emotional stimulus features without establishing a task set involving stimulus valence, which could directly interfere with the processing of happy and fearful facial emotional expressions. In order to capture the time course of task set activation and inhibition, we also varied the time interval between the response to the induction task and the onset of prime presentation, response-prime interval (RPI). The taskswitching literature (Rogers \& Monsell, 1995) as well as previous work using the induction task paradigm (Kiefer, 2018) suggest that a task representation is active for about $600 \mathrm{~ms}$ after task completion, but inhibited thereafter (Mayr \& Keele, 2000). Task sets should therefore only be active shortly after completing the induction task at a RPI of $200 \mathrm{~ms}$, but should be inhibited at a longer RPI of $800 \mathrm{~ms}$ (Kiefer \& Martens, 2010) when participants are prepared to execute the upcoming gender decision task.

If subliminal gender priming of faces depends on a subcortical route, priming effects should not be moderated by the induction task manipulation at neither RPI. However, if gender priming at least partially depends on cortical visual processing, e.g. on visual shape analysis, gender priming should be larger following the perceptual shape induction task than following the emotional arousal induction task at the short RPI. Furthermore, we expected gender priming effects to be moderated by emotional congruency, in particular following the emotional induction task at the short RPI, when (cortical) emotional pathways are sensitized. In accordance with the findings by Schmitz and Wentura (Schmitz \& Wentura, 2012; Schmitz, et al., 2014) described above, we assumed that prime and target faces with congruent emotional expressions would facilitate each other, thereby increasing the magnitude of the gender priming effect. As analyses of emotional expressions might be boosted when the attentional focus rests on emotional arousal rather than perceptual shape, we expected this interaction between gender and emotional congruency to be more pronounced following the emotional than following the perceptual induction task at the short RPI.

At the long RPI, when the task sets of the induction tasks are inhibited in preparation for the upcoming gender decision task, priming should be larger following emotional than following perceptual induction. 
This reversal of priming effects is expected, because the inhibited task set of the perceptual induction task at the long RPI should attenuate subliminal gender processing, whereas the inhibited emotional task set is neutral with regard to gender processing. Moreover, due to the inhibition of the emotional task set at the long RPI, the interaction between emotional and gender congruency following emotional induction, as predicted for the short RPI, should be absent at the long RPI.

\section{Methods and Materials}

\subsection{Participants}

Thirty-two healthy, right-handed (according to Oldfield, 1971) native German speakers with normal or corrected-to-normal vision participated in the study. Data of seven participants had to be rejected, because their performance in the subliminal prime identification test exceeded the $95 \%$ confidence interval of chance performance (> $65 \%$ correct) calculated by approximation through the normal distribution (Kiefer, Liegel, Zovko, \& Wentura, 2017), and data from one participant was excluded because of excessive errors. The remaining 24 participants (male/female: 13/11) were in the age range of 19-27 years, with a mean age of 22.6 years. We aimed to collect data from 24 valid cases in order to obtain a sample size comparable to earlier studies using the induction task paradigm (range 20-22, Kiefer \& Martens, 2010; Martens et al., 2011). When taking the effect size of the theoretically most relevant triple interaction between semantic relatedness, induction task and RPI $(\mathrm{dz}=0.74)$ in Exp. 3 of the Kiefer and Martens (2010) paper as an estimate for the calculation of the sample size in a power analysis with $G^{\star}$ Power (Faul et al., 2007), a sample of 22 participants is required to achieve a power (1-beta) of 0.95 at an alpha error level of $p<0.05$. Hence, the sample size of the present experiment $(\mathrm{N}=24)$ provides sufficient statistical power to detect the predicted three-way interaction. Participants were not informed about the presence of the masked primes before the main experiment, but were debriefed thereafter. All participants were naive to the purpose of the study. This study was carried out in accordance with the recommendations of the Declaration of Helsinki. The protocol was approved by the Ethics Committee of Ulm University. All subjects gave written informed consent in accordance with the Declaration of Helsinki. The participants were paid 17 euros or received course credit for participation.

\subsection{Materials}

\subsubsection{Induction tasks}

For the emotional arousal decision induction task, 64 color pictures were selected from the International Affective Picture System (IAPS Lang \& Bradley, 2007). These pictures were selected according to the IAPS database, which comprises arousal and emotional valence ratings on a 9 point Likert-scale. Thirty-two color pictures with high arousal $(M=6.43, S E=0.10)$ and 32 pictures with low arousal $(M=3.15, S E=0.10)$ were chosen, which were repeated twice during the emotional induction task for each participant. The set of pictures for the high arousal condition consisted of 16 pictures with positive emotional valence $(M=7.20, S E$ $=0.13)$ and another 16 pictures with negative emotional valence $(M=2.85, S E=0.13)$, whereas all pictures for the low arousal condition were of neutral emotional valence $(M=5.03, S E=0.09)$. Importantly, positive and negative pictures of the high arousal condition were matched with regard to arousal ratings, so that the positive $(M=6.46, S E=0.11)$ and negative $(M=6.41, S E=0.11)$ pictures did not differ from each other in arousal $(F(1,32)=0.063, p=0.80)$.

In the perceptual shape decision induction task, 64 color pictures of living or nonliving objects were used: Half of them depicted a round and the other half an elongated object. Object pictures were balanced across living and nonliving object categories to exclude confounding effects. The same stimuli were previously used to investigate modulation of masked semantic or visuo-motor priming by task sets (Kiefer \& Martens, 2010; Zovko \& Kiefer, 2013). 


\subsubsection{Primed gender decision task}

For the subliminal gender priming task, 64 colored facial pictures were selected from the Karolinska Directed Emotional Faces (KDEF) database (Lundqvist, Flykt, \& Öhman, 1998). These facial pictures showed 64 different individuals (male/female: 32/32). Individuals were depicted from a front view. Half of them (male/female: 16/16) showed a positive facial expression (happy), the other half (male/female: 16/16) a negative facial expression (fearful). Sixteen positive (male/female: 8/8) and 16 negative (male/female: $8 / 8$ ) faces of them were used as primes, another 16 positive (male/female: $8 / 8$ ) and 16 negative faces (male/ female: $8 / 8$ ) were used as targets. Hence, prime faces did not appear as targets. Prime and target faces were combined such that there were 32 emotionally congruent pairings (positive-positive, negative-negative) and 32 emotionally incongruent pairings (positive-negative, negative-positive). All prime-target pairs were then balanced by gender, so that male and female facial pictures were equally often used in each task condition. Altogether, there were four different prime-target conditions in the primed gender decision task: gender congruent + emotion congruent vs. gender incongruent + emotion congruent vs. gender congruent + emotion incongruent vs. gender incongruent + emotion incongruent. In turn, each condition comprised eight different pairs of prime and target faces. Each prime-target pair was finally presented twice in emotionally congruent and incongruent conditions in each induction task (altogether 8 repetitions). Thus, the stimuli in the congruent and incongruent conditions were identical, but only differentially paired. For that reason, unspecific visual differences between congruency conditions can be excluded.

Additionally, eight scrambled pictures composed of small parts of various complex visual scenes served as forward and backward masks. These masking stimuli have been successfully used in a previous priming study (Kiefer, Sim, \& Wentura, 2015). The masks were selected randomly.

A between-subject ANOVA on arousal scores based on the KDEF database (Goeleven, De Raedt, Leyman, \& Verschuere, 2008) with the factors emotional expression (positive vs. negative) and type of stimuli (primes and target) did not yield significantly different arousal scores between faces with positive and negative expressions $(F(1,60)=1.87, p=0.18)$ as well as between the primes and targets $(F(1,60)=1.73$, $p=0.19)$. Thus, the positive $(\mathrm{M}=3.77, S E=0.07)$ and negative $(\mathrm{M}=3.63, S E=0.07)$ faces as well as prime (M $=3.63, S E=0.07)$ and target faces $(M=3.76, S E=0.07)$ were comparable with regard to arousal.

\subsection{Procedure}

The total number of 256 trials was presented in four blocks of 64 trials each, separated by breaks. The trials of the induction tasks and the primed gender decision task were combined such that all conditions of the induction task and the primed gender decision task occurred equally often and were entirely balanced. For that reason, only attentional sets established by the induction tasks and not stimulus or response related processes systematically influenced subsequent gender priming effects. The order of trials within each block was randomized in each subject. Each trial started with three hash marks (\#\#\#), which prompted the participant to initiate the following sequence of events with a button press: First, a task cue signaling the type of induction task to be performed was presented for $750 \mathrm{~ms}$ (written words "Form" [engl. shape] for the perceptual induction task or "Erregung" [engl. arousal] for the emotional induction task). Thereafter a fixation cross was shown for $750 \mathrm{~ms}$, followed by an induction task picture for $500 \mathrm{~ms}$. In the perceptual induction task, participants were instructed to decide whether the depicted object had a round or elongated shape. In the emotional induction task, participants had to decide whether the picture showed an emotionally arousing or a neutral scene. Responses in the induction task were given via key presses with left and right index fingers. Instructions emphasized both response speed and accuracy. A black screen appeared until the participants gave their response. After responding to the induction task, the forward mask appeared for either $200 \mathrm{~ms}$ (short RPI condition) or $800 \mathrm{~ms}$ (long RPI condition) in half of the trials each, followed by the facial prime picture for $32.6 \mathrm{~ms}$ and the backward mask (32.6 ms). As in previous work (Kiefer \& Martens, 2010), this RPI variation was used to track the temporal dynamics of induction task effects on subsequent priming. After the backward mask, a target stimulus was presented for $200 \mathrm{~ms}$, followed by a black screen until the participant gave a response (see fig 1). Participants had to decide as 
quickly and accurately as possible whether or not the target was a male or female face (gender decision task). They provided their decision by pressing one of two response buttons with the left and right index fingers, respectively. Assignment of the response categories in the induction task and the gender decision task to right hand or left hand key presses were counterbalanced across participants. All induction pictures (low arousing [i.e. neutral], high arousing [i.e. positive, negative] for the emotional induction task as well as the long and elongated pictures for the perceptual induction task) were assigned to the conditions of the priming task in a counterbalanced fashion. Hence, there was no systematic relation between the targets of the induction tasks and the stimuli of the gender decision task. Altogether, there were 256 trials within 2 induction tasks (perceptual vs. emotional) x 2 prime-target congruency of emotion (emotional congruent vs. incongruent) x 2 prime-target congruency of gender (gender-congruent vs. incongruent) x RPI (short vs. long) design. Hence, each of these experimental conditions is comprised of 16 trials.

All pictures (including masks) had a size of 170 pixels in width and of 216 pixels in height and were presented in the center of the computer monitor. The viewing angle for the pictures subtended about $4.3^{\circ}$ horizontally and $5.4^{\circ}$ vertically from a viewing distance of $80 \mathrm{~cm}$. The stimuli were displayed on a black background synchronously with the CRT monitor (19”, ADI MicroScan, ADI Systems Inc., Chicago, IL, USA) at a refresh rate of $16.67 \mathrm{~ms}$. Prior to the experiment, all participants received task instructions verbally and in written form and practiced the induction task and the gender decision task, with 16 trials each. Subsequently, they practiced the tasks in combination in the same sequence as in the main experiment. The experiment was run using the Experimental Runtime System software (BeriSoft cooperation, Frankfurt, Germany) under MS DOS.

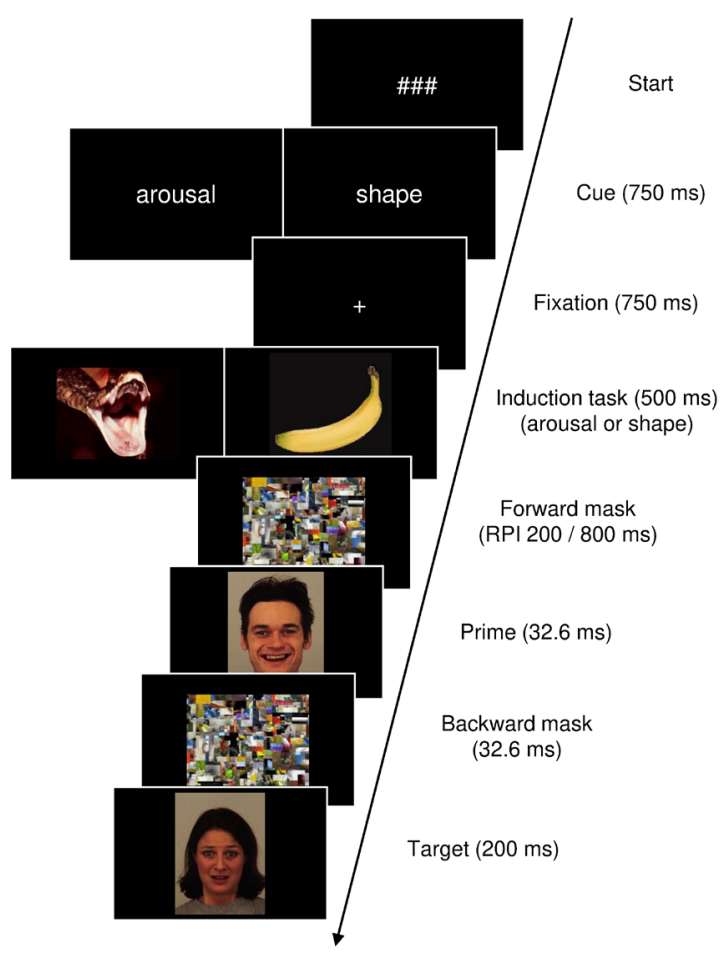

Subliminal gender priming task

Figure 1: Trial sequence in the subliminal gender priming task. At the start of each trial, a perceptual or emotional induction task was performed to establish an attentional focus on shape vs. arousal. In the induction tasks, participants had to classify the picture either according to its shape (perceptual induction task) or its arousal (emotional induction task). Thereafter, the masked prime face and the target face appeared. The gender of the target faces that had to be reported by a left or right index finger button press in both experiments. The masked face prime was presented either $200 \mathrm{~ms}$ (short response prime interval, $\mathrm{RPI}$ ) or $800 \mathrm{~ms}$ (long RPI) following the response to the induction task. The "+" sign indicates the fixation cross. The hash marks indicate the breaks between the trials. 
After the main experiment, participants were informed about the presence of the prime between the masks and were asked whether they have recognized that primes had been presented. None of the participants reported awareness of the primes. Thereafter, an objective measure of prime identification was obtained (Kiefer, 2002) using an identical trial structure and the same condition combinations as in the main experiment. The prime identification test is comprised of 96 trials. To obtain a liberal estimate of masked prime identification, we only used data from trials with the long RPI, because the masking influence of the induction word was reduced in these trials. However, unlike in the main experiment, participants had to perform the gender decision on the subliminal primes between the masks, while stressing accuracy over response speed. Participants were also instructed to guess when they were not able to consciously recognize the masked primes. Only data of participants, who were not able to identify the masked prime stimulus above chance level, were included in the analysis (performance within the $95 \%$ confidence interval of chance level, calculated by approximation through the normal distribution, > $65 \%$ correct responses).

\section{Results}

\subsection{General}

For the analyses of reaction times (RT), mean correct RT were calculated for each condition. Responses faster or slower than two standard deviations of the individuals' means were defined as outliers and not entered into data analysis $(\mathrm{M}=7.86 \%)$.

\subsection{Induction tasks}

Perceptual (shape decision) and emotional (emotional arousal decision) induction tasks had a differential difficulty level with regard to reaction times and error rates. In a two-tailed paired $t$-test, reaction time and error rates significantly differed between the emotional (arousal) and the perceptual (shape) decision task (for reaction time $\mathrm{M}=597 \mathrm{~ms}$ emotional versus $500 \mathrm{~ms}$ shape, $t(23)=-14.83, p<0.01$; for error rates $\mathrm{M}=$ $10.68 \%$ emotional versus $4.24 \%, t(23)=-7.68$ shape, $p<0.01)$. Reactions to trials in the perceptual induction task were faster compared to those in the emotional induction task. Analyses of ER revealed a higher ER for emotional arousal decisions than for perceptual shape decisions.

\subsection{Primed gender decision task}

A repeated-measures analysis of variance (ANOVA) on mean correct RTs with the within-subject factors induction task (perceptual vs. emotional), RPI (short vs. long), prime-target congruency of emotion (emotional congruent vs. incongruent) and prime-target congruency of gender (gender-congruent vs. gender-incongruent) was performed. The analyses showed significant main effects of induction task $(F(1,23)=6.008, p<0.02)$, RPI $(F(1,23)=72.94, p<0.01)$ and gender congruency $(F(1,23)=19.02, p<0.01)$. Gender decisions were faster in the perceptual induction task condition $(\mathrm{M}=504 \mathrm{~ms}, S E=8.14)$ than in the emotional induction task condition $(\mathrm{M}=508 \mathrm{~ms}, S E=8.14)$. The reactions were faster in long RPIs $(M=493 \mathrm{~ms}, S E=7.99)$ than in short RPIs $(M=519 \mathrm{~ms}, S E=8.58)$. We also found the expected gender congruency effect: Reactions in gender-congruent trials $(M=500 \mathrm{~ms}, S E=8.55)$ were faster than in genderincongruent trials $(\mathrm{M}=512 \mathrm{~ms}, S E=7.98)$. Most importantly, there was a three-way interaction between induction task, gender congruency and emotional congruency $(F(1,23)=6.76, p=0.02)$. The main effect of emotional congruency was not significant $(F(1,23)=2.32, p=0.14)$. No other combination of factors yielded a significant effect (all $p s>0.10$ ).

In order to further explore this interaction between emotional and gender congruency, we performed two separate repeated-measures ANOVAs in the emotional and perceptual induction task conditions with the factors RPI, gender congruency and emotional congruency. Following perceptual induction task trials, 
the main effects of RPI and gender congruency were significant $(F(1,23)=36.87$ for RPI, $F(1,23)=17.53$ for gender congruency, all $p s<0.01)$ : Gender decisions were faster in the gender congruent $(\mathrm{M}=497 \mathrm{~ms}, S E$ $=8.26)$ than in the incongruent condition $(\mathrm{M}=511 \mathrm{~ms}, S E=8.37)$. Reactions in the gender decision task were faster at long RPIs $(M=492 \mathrm{~ms}, S E=8.28)$ than at short RPIs $(M=515 \mathrm{~ms}, S E=8.46)$. In particular, we found a significant interaction between emotional congruency and gender congruency $(F(1,23)=5.49$, $p<0.03$ ). Subsequent Fisher's LSD post-hoc tests hereby revealed a significant gender priming effect ( $p=$ 0.001 ) only for the emotionally congruent prime-target pairs (see fig 2 left). Here, gender-congruent trials showed shorter reaction times $(\mathrm{M}=495 \mathrm{~ms}, S E=9.03)$ than gender incongruent trials $(\mathrm{M}=517 \mathrm{~ms}, S E=9.87)$, whereas the gender priming effect in emotional incongruent prime-target pairs was not significant. $(p=$ 0.23). Following emotional induction task trials, only significant main effects of gender congruency $(F(1,23)$ $=6.69, p<0.02)$ and $\operatorname{RPI}(F(1,23)=60.29, p=0.001)$ were found: Gender decisions were faster in the gender congruent $(\mathrm{M}=503 \mathrm{~ms}, S E=9.10)$ than in the gender incongruent conditions $(\mathrm{M}=513 \mathrm{~ms}, S E=7.84)$ (see fig 2 right). Reactions in the gender decision task were again faster at long RPIs (M = $494.94 \mathrm{~ms}, S E=7.90)$ than at short RPIs $(M=522 \mathrm{~ms}, S E=8.98)$. No further significant effects were found ( $p s>0.10)$. An analogue analysis of ER yielded only a main effect of gender congruency $(F(1,23)=9,79, p=0.005)$. Gender decisions were more error-prone in gender incongruent $(\mathrm{M}=5.21 \%, S E=0.62)$ than in gender congruent trials $(\mathrm{M}=$ $2.93 \%, S E=0.62)$.

\section{Subliminal gender priming task}

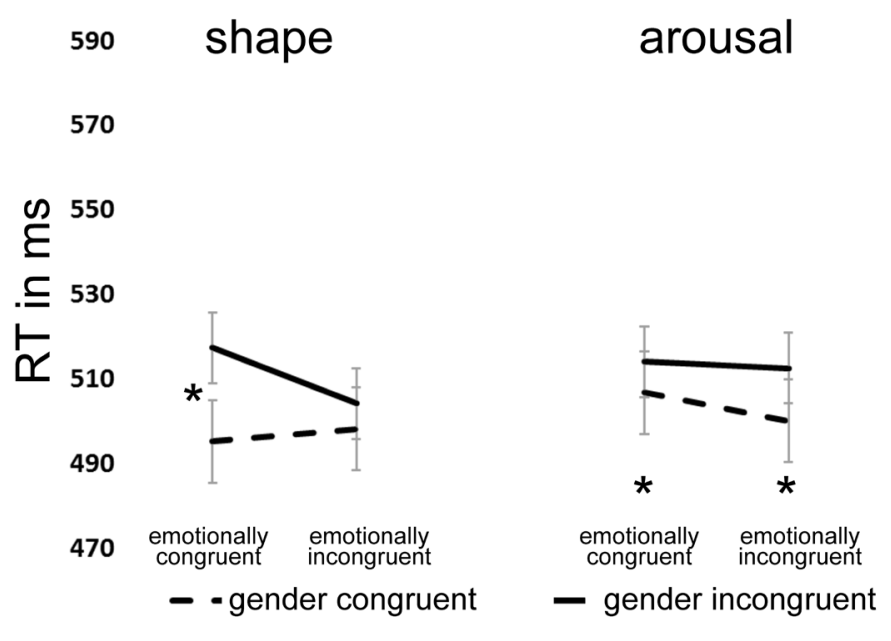

Figure 2. Gender decision mean latencies as a function of gender congruency, emotional congruency, induction task in the subliminal gender priming task. Gender priming effects are highlighted as significant with an asterisk ( $\left.{ }^{*}\right)$. The vertical lines depict the standard error of means of each condition. Shape: shape decision, perceptual induction task; arousal: arousal decision, emotional induction task condition.

In the masked prime identification test, prime identification accuracy did not significantly deviate from the chance level of $50 \%(\mathrm{M}=50.29 \%, t(23)=0.38, p=0.71)$, which is to be expected as a consequence of mere guessing. Furthermore, we calculated $d^{\prime}$ measures according to signal detection theory (Green \& Swets, 1966) for masked prime identification from hit and false alarm rates. For each participant, relative frequency of correct responses to female prime faces were treated as hit rate, whereas erroneous responses to male prime faces were entered as false alarm rate. A two-tailed paired t-test showed that $\mathrm{d}^{\prime}$ did not significantly deviate from zero (mean $\mathrm{d}^{\prime}=0.206, t(23)=1.69, p=0.10$ ). Hence, masking successfully prevented participants from conscious prime identification. Furthermore, we tested whether individual prime visibility ( $\left.\mathrm{d}^{\prime}\right)$ and individual gender congruency effects (RT difference incongruent minus congruent) were correlated by calculating Pearson's correlation coefficients. Correlations between prime visibility and 
gender congruency effects were small and not significant, neither for gender congruency effects pooled across induction tasks $(r=-0.15, p=0.47)$ nor for congruency effects calculated separately for perceptual $(r$ $=-0.19, p=0.37)$ and emotional induction tasks $(r=-0.05, p=0.80)$.

\section{Discussion}

The present study investigated attentional control effects associated with activated task sets on the processing of gender and emotional expression of faces under subliminal viewing conditions. Processing of gender and emotional expression of faces was probed by a face priming paradigm during a gender decision task by orthogonally varying gender and emotional expression congruency of facial prime-target pairs. Prior to the prime presentation, different attentional control settings were established by shape decision vs. arousal decision tasks, which served to activate a perceptual vs. an emotional attentional focus. Unlike subliminal word or object priming (Kiefer, 2018; Kiefer, Adams, \& Zovko, 2012; Martens, et al., 2011), previous research indicated that subliminal face priming during a gender decision task does not require attentional amplification (Finkbeiner \& Palermo, 2009; Khalid, et al., 2013). However, the influence of feature-specific attention on subliminal face priming has not been previously investigated. The present research therefore asked, whether manipulating the attentional focus by perceptual vs. emotional induction tasks has the power to modulate subsequent subliminal face priming.

We found overall faster reaction times in the gender decision task for gender-congruent prime-target faces compared to gender-incongruent prime-target pairings (i.e., a gender priming effect). Hence, in line with previous research (Finkbeiner \& Palermo, 2009; Khalid, et al., 2013), our study indicates that gender information of faces can also be processed at an unconscious (subliminal) level. In contrast, task-irrelevant emotional expression congruency (i.e. prime and target faces showed the same emotional expression) did not evoke a general priming effect.

\section{Absence of emotional priming effects}

Induction tasks significantly influenced subliminal gender priming effects as a function of emotional expression congruency. After the perceptual induction task, subliminal gender priming was only significant for emotionally congruent prime-target pairs, but not for emotionally incongruent pairings. In contrast, after the emotional induction task, subliminal gender priming was observed irrespective of emotional congruency (though at a descriptive level, gender priming was smaller for emotionally congruent pairings). The differences between emotionally congruent and incongruent conditions were not significant after neither induction task. This absence of a general emotional congruency effect in the gender decision task under subliminal viewing conditions is in line with earlier research demonstrating processing of unconscious information, only when it is congruent with current task representations (Ansorge \& Neumann, 2005; Eckstein \& Perrig, 2007). However, in contrast to our expectations, the emotional induction task did not boost subliminal processing of task-irrelevant emotional facial expressions during gender decisions and did not increase subliminal emotional expression priming. We would like to add at this place that we ran the same subliminal face priming paradigm using an emotional expression decision task (happy vs. fearful facial expression) instead of the gender decision task (unpublished data). Although in this experiment emotional facial expressions were task-relevant, we nevertheless failed to observe a significant emotional priming effect subsequent to either induction task condition. In line with this unpublished result, previous studies also failed to find subliminal priming effects with fearful faces in an emotion decision task, even when emotional facial expression was task-relevant (Hedger, Adams, \& Garner, 2015; Khalid \& Ansorge, 2017). Emotional expressions seem to facilitate responses to congruent targets only when processed at a conscious level (Khalid \& Ansorge, 2017). As we only tested unawareness of facial gender information, but not unawareness of facial emotional information, strictly speaking, the invisibility of emotional expression is in question. However, the study using the emotional expression decision task (unpublished data), mentioned above, indicated that, with the present stimulus material, emotional expression decisions were 
more difficult than gender decisions. Due to the difficulty of emotional expression discrimination, it is not very likely that participants were unaware of facial gender information, but were aware of facial emotional expression.

Another possible limitation with regard to emotional congruency effects should be considered: As we only used two emotional facial expressions with positive vs. negative valence (happy vs. fearful) to form prime-target pairs of congruent and incongruent emotional expressions, the emotion and the valence dimensions were fully confounded in our study. It must, therefore, remain open, whether congruency of emotion, congruency of valence or the combination of both are the relevant factors for constituting the relation of emotionally congruent prime-target pairings.

It could also be argued that the missing emotional congruency effect subsequent to the emotional induction task could be due to a much stronger priming effect of the clearly visible targets of the induction task on the valence of the targets in the priming task. However, in our study design, all induction pictures (emotional as well as neutral pictures) were assigned to the conditions of the priming task in a counterbalanced fashion. Hence, there was no fixed relation between the targets of the induction tasks and the stimuli of the face priming task. For that reason, valence of the induction task stimuli could not influence emotional congruency effects systematically. Moreover, even in the perceptual induction task, in which the stimuli of the induction task were emotionally neutral, emotional congruency effects were absent. Finally, we conducted fine-grained analyses of congruency effects subsequent high arousing positive and negative induction task pictures as well as subsequent low arousing neutral induction task pictures. This analysis did not reveal differential congruency effects indicating that valence or arousal of the targets in the induction tasks did not systematically influence emotional congruency effects.

The absence of the expected emotional priming effect under emotional induction could possibly be due to the higher difficulty of the emotional induction task (arousal decision) compared to the perceptual induction task (shape decision) as indexed by slower responses and increased error rates. We outlined in the introduction section that unconscious processing at a cortical level presumably depends on attentional resources (Finkbeiner \& Palermo, 2009; Kiefer \& Brendel, 2006). The attentional focus on emotional stimulus features established under emotional induction might have been not sufficient to boost the emotional priming effects based on expression congruency because general attentional resources were reduced in this condition. However, we hasten to add at this place that the subliminal gender priming effect was not abolished under emotional induction indicating that attentional resources were sufficient at least for unconscious processing of gender information.

Another aspect is that ratings of all emotional stimuli, in particular of the faces, were taken from the respective databases, e.g. KDEF database (Goeleven et al., 2008), and were not confirmed by the participants of the study. It was important for us to determine the properties of the stimuli in advance, in order to create the different experimental conditions. Of course, additional ratings of our participants as a treatment check would have been valuable. The absence of emotional congruency effects could in principle be due to a differential emotional evaluation of the stimuli of our participants compared with the norming sample. However, it is difficult to perform a meaningful analysis with personalized ratings.

\section{Interaction between emotional congruency and gender congruency}

Although we did not observe emotional priming effects in our study, emotional congruency moderated the gender priming effect specifically subsequent to the perceptual induction task. This indicates that a perceptual task set enhances processing of emotional facial expressions, which in turn influences the magnitude of gender priming effects: Gender priming effects were only found for prime target pairs with emotionally congruent facial expressions, but were absent for pairs with emotionally incongruent expressions. This interaction between gender and emotional congruency is in line with earlier findings by Schmitz and Wentura (2012; see also Schmitz, et al., 2014). Similar to our results, they found larger effects of semantic congruency for evaluatively congruent than for evaluatively incongruent prime-target pairs in a flanker task. These interactions between congruency effects across stimulus dimensions (emotion vs. gender/semantics) are in line with the three-process model of evaluative priming (Schmitz, et al., 2014; 
Wentura \& Rothermund, 2003). Although developed to explain evaluative and not gender priming, this model is a suitable theoretical framework to interpret the present findings. This model assumes a parallel activation of prime and target representation, a mutual facilitation between primes and targets and finally response related processes. According to this model, emotionally congruent prime target pairings mutually facilitate each other, leading to increased prime activation maintenance and subsequently to larger gender priming. As this interaction between gender and emotional congruency was only observed subsequent to perceptual induction, this mutual facilitation of emotionally congruent prime-target expressions - at least in our experimental setting - seem to be based on perceptual processing of the subliminal prime face, but not on emotional semantic processing. With pictorial facial stimuli, mutual facilitation of emotionally congruent prime-target representations might depend on similarity of their visual configuration (Tanaka, Kiefer, \& Bukach, 2004), coding the spatial relation of parts such as mouth, nose and eyes, and not on their emotional semantic content. A perceptual (and not a semantic) basis of the analysis of emotional congruency has also been found for pictorial stimuli other than faces. Similar to the present results, subliminal evaluative priming of pictures of visual scenes in an evaluative decision task has been found to depend on an attentional focus on perceptual shape, but not on semantics (Kiefer, et al., 2017).

Comparably to earlier studies using the induction task paradigm (e. g., Kiefer \& Martens, 2010), a different set of stimuli was used for the alternative induction task conditions. For that reason, with such a study design, stimuli themselves and not only the task set at hand might influence subsequent priming. In the present study and the earlier studies, we avoided at least that stimuli within each set had a systematic effect on priming by assigning them to the different priming conditions in a counterbalanced fashion (see also the discussion above). The advantage of using the same stimuli and the alternative tasks is obvious: Any differences in subsequent priming can unequivocally relate to the task. However, the usage of the same stimuli in the alternative induction tasks has also several disadvantages, which motivated us to use different stimuli: First of all, it is difficult to find a relatively large set of stimuli, which can be used in alternative types of induction tasks (e.g., arousal vs. shape decision). Secondly and even more importantly, when the same stimuli are used, stimuli have to be repeatedly represented under different task instructions. In addition to unspecific repetition effects, presenting the same set of stimuli under different task instructions might compromise straightforward activation of the appropriate task set and thus impair attentional sensitization of processing pathways. Presentation of a stimulus under a given task most likely leads to an association of the stimulus with the task set and the corresponding response (Damian, 2001; Kiefer, et al., 2015). These associations have to be inhibited at second presentation under a different task instruction with presumably negative consequences on the speed of task set activation and subsequent attentional carry-over effects on masked priming due to task switching (Kiesel et al., 2010). Given these considerations regarding advantages and disadvantages of each type of study design, we decided to use different stimuli in the alternative induction task conditions.

Variation of the interval between the response in the induction task and the prime (RPI) did not significantly modulate priming effects after neither induction task. Previous research frequently observed a suppression of the task set, when the task set of the induction task has been completed for $500 \mathrm{~ms}$ and longer (Kiefer, 2018; Kiefer \& Martens, 2010; Martens \& Kiefer, 2009). In these earlier studies, the induction task included pictures as stimuli and the priming task words. An absence of RPI effects on priming effects following different induction tasks has been already observed in a previous study with a pictorial response priming paradigm and the same type of pictorial induction task (Martens, et al., 2011). Possibly, due to the pictorial nature of the induction task and the priming task, the task set of the induction task remained active at both RPIs and did not need to be suppressed after about $500 \mathrm{~ms}$ in preparation for the upcoming priming task. Overall, we found faster reactions at long than at short RPIs. This general RPI effect most likely reflects the fact that participants have more time to implement the task set of the gender decision task after completing the induction task as it is typical observed in task switching research (Kiesel, et al., 2010). 


\section{Implications for the automaticity of processing emotional expression and gender information}

In line with the attentional sensitization model of unconscious cognition (Kiefer \& Martens, 2010), unconscious processing of emotional expression was influenced by the attentional focus established by the induction tasks, which in turn moderated the magnitude of the subliminal gender priming effect. This indicates that unconscious processing of emotional facial expression is susceptible to attentional control in congruency with the well-documented attentional effects on subliminal word and object processing (for reviews, see Ansorge, et al., 2014; Kiefer, 2018). However, besides the moderation of subliminal gender priming by emotional congruency following perceptual induction, the magnitude of subliminal gender priming was not generally influenced by the previously performed induction tasks. In particular, subliminal gender priming was obtained following both perceptual and emotional induction tasks. This indicates that unconscious processing of facial gender information is largely unaffected by feature-specific attention (here: attentional focus on perceptual vs. emotional information). In particular, gender priming was not abolished by directing attention to emotional stimulus features, which are not relevant for determining the gender of a face. The present results are thus in line with previous research demonstrating subliminal gender priming effects irrespective of whether the masked prime faces were attended or not (Finkbeiner \& Palermo, 2009; Khalid, et al., 2013).

Overall, processes underlying subliminal gender priming seem to be largely congruent with the classical theoretical stance of automaticity (Posner \& Snyder, 1975; Schneider \& Shiffrin, 1977) assuming automatic processing to be independent from cognitive control influences. As we did not use filtered images containing only high-frequency or low-frequency visual information, we cannot determine whether subliminal gender priming depends on low frequency information processed along the subcortical magno-cellular pathway. Previous results, however, suggest that processing facial low frequency information along the subcortical route might not be influenced by spatial attention (Khalid, et al., 2013).

Nevertheless, the moderation of gender priming by emotional congruency under perceptual induction indicates an indirect influence of attention through its influence on processing emotional expression. One may speculate that this interaction between gender and emotional congruency arises from cortical face processing, in which unconscious visual representations of expression congruent faces is enhanced through mutual facilitation of prime and target face representations. As the present gender priming task can be considered as a response priming paradigm (Khalid \& Ansorge, 2017), in which priming arises at the level of response preparation, we assume that information from both cortical and subcortical processing of the subliminal prime faces reaches the motor system and facilitates or interferes with the response to the visible target depending on gender congruency.

In conclusion, the present study shows that subliminal gender priming does not depend on a featurespecific attentional focus on perceptual shape features, but is also observed when attention is directed to priming-irrelevant emotional stimulus features. However, subliminal gender priming is moderated by the congruency of emotional facial expressions, when attention is directed to perceptual shape. Most likely, attention to shape facilitates subliminal processing of task-irrelevant emotional facial expressions. Mutual facilitation of emotionally congruent prime and target representations, in turn, enhances the gender priming effect compared with emotionally incongruent pairings.

Ethics approval and consent to participate: This study was carried out in accordance with the recommendations of the Declaration of Helsinki. The protocol was approved by the Ethics Committee of Ulm University. All subjects gave written informed consent in accordance with the Declaration of Helsinki.

Human and animal rights: Rights of the human participants were protected by following the Declaration of Helsinki. The protocol of the study was approved by the Ethics Committee of Ulm University.

Consent for publication: Not applicable. 
Conflict of interest: The authors declare no conflict of interest, financial or otherwise.

Financial Support: This research received no specific grant from any funding agency, commercial or nonprofit sectors.

\section{References}

Ansorge, U., Kunde, W., \& Kiefer, M. (2014). Unconscious vision and executive control: How unconscious processing and conscious action control interact. Consciousness and Cognition, 27, 268-287.

Ansorge, U., \& Neumann, O. (2005). Intentions determine the effect of invisible metacontrast-masked primes: Evidence for top-down contingencies in a peripheral cuing task. Journal of Experimental Psychology: Human Perception and Performance, 31(4), 762-777. doi: 10.1037/0096-1523.31.4.762

Damian, M. F. (2001). Congruity effects evoked by subliminally presented primes: Automaticity rather than semantic processing. Journal of Experimental Psychology: Human Perception \& Performance, 27(1), 154-165.

de Gelder, B., Vroomen, J., Pourtois, G., \& Weiskrantz, L. (1999). Non-conscious recognition of affect in the absence of striate cortex. Neuroreport, 10(18), 3759-3763.

Dell'Acqua, R., \& Grainger, J. (1999). Unconscious semantic priming from pictures. Cognition, 73(1), B1-B15.

Dimberg, U., \& Petterson, M. (2000). Facial reactions to happy and angry facial expressions: Evidence for right hemisphere dominance. Psychophysiology, 37(5), 693-696.

Draine, S. C., \& Greenwald, A. G. (1998). Replicable unconscious semantic priming. Journal of Experimental Psychology: General, 127(3), 286-303.

Duckworth, K. L., Bargh, J. A., Garcia, M., \& Chaiken, S. (2002). The automatic evaluation of novel stimuli. Psychological Science, 13(6), 513-519. doi: 10.1111/1467-9280.00490

Eckstein, D., \& Perrig, W. J. (2007). The influence of intention on masked priming: A study with semantic classification of words. Cognition, 104(2), 345-376. doi: 10.1016/j.cognition.2006.07.005

Finkbeiner, M., \& Palermo, R. (2009). The role of spatial attention in nonconscious processing: A comparison of face and nonface stimuli. Psychological Science, 20(1), 42-51.

Green, D., \& Swets, J. (1966). Signal detection theory and psychophysics Wiley.[arLEK, DDD. NAM, MT].

Goeleven, E., De Raedt, R., Leyman, L., \& Verschuere, B. (2008). The Karolinska directed emotional faces: a validation study. Cognition and emotion, 22(6), 1094-1118.

Hedger, N., Adams, W. J., \& Garner, M. (2015). Fearful faces have a sensory advantage in the competition for awareness. Journal of Experimental Psychology: Human Perception and Performance,41(6), 1748-1757.

Johnson, M. H. (2005). Subcortical face processing. Nature reviews. Neuroscience, 6(10), 766-774. doi: 10.1038/nrn1766

Jolij, J., \& Lamme, V. A. (2005). Repression of unconscious information by conscious processing: Evidence from affective blindsight induced by transcranial magnetic stimulation. Proceedings of the National Academy of Sciences of the United States of America, 102(30), 10747-10751.

Khalid, S., \& Ansorge, U. (2017). Subliminal face emotion processing: A comparison of fearful and disgusted faces. Frontiers in Psychology, 8, 1028-1046.

Khalid, S., Finkbeiner, M., Konig, P., \& Ansorge, U. (2013). Subcortical human face processing? Evidence from masked priming. Journal of Experimental Psychology: Human Perception and Performance, 39(4), 989-1002. doi: 10.1037/a0030867

Kiefer, M. (2002). The $\mathrm{N} 400$ is modulated by unconsciously perceived masked words: Further evidence for an automatic spreading activation account of N400 priming effects. Cognitive Brain Research, 13(1), 27-39.

Kiefer, M. (2012). Executive control over unconscious cognition: Attentional sensitization of unconscious information processing. Frontiers in Human Neuroscience, 6, 61. doi: https://doi.org/10.3389/fnhum.2012.00061

Kiefer, M. (2018). Cognitive control over unconscious cognition: Flexibility and generalizability of task set influences on subsequent masked semantic priming. Psychological Research, 83(7), 1556-1570. doi: 10.1007/s00426-018-1011-x.

Kiefer, M., Adams, S. C., \& Zovko, M. (2012). Attentional sensitization of unconscious visual processing: Top-down influences on masked priming. Advances in Cognitive Psychology, 8(1), 50-61.

Kiefer, M., \& Brendel, D. (2006). Attentional modulation of unconscious 'automatic' processes: Evidence from event-related potentials in a masked priming paradigm. Journal of Cognitive Neuroscience, 18(2), 184-198.

Kiefer, M., Liegel, N., Zovko, M., \& Wentura, D. (2017). Mechanisms of masked evaluative priming: Task sets modulate behavioral and electrophysiological priming for picture and words differentially. Social cognitive and Affective Neuroscience, 12(4), 596-608. doi: 10.1093/scan/nsw167

Kiefer, M., \& Martens, U. (2010). Attentional sensitization of unconscious cognition: Task sets modulate subsequent masked semantic priming. Journal of Experimental Psychology: General, 139(3), 464-489. doi: 10.1037/a0019561

Kiefer, M., Sim, E. J., \& Wentura, D. (2015). Boundary conditions for the influence of unfamiliar non-target primes in unconscious evaluative priming: The moderating role of attentional task sets. Consciousness and Cognition, 35, 342-356. doi: 10.1016/j.concog.2015.01.010 
Kiefer, M., \& Spitzer, M. (2000). Time course of conscious and unconscious semantic brain activation. Neuroreport, 11(11), 2401-2407.

Kiesel, A., Steinhauser, M., Wendt, M., Falkenstein, M., Jost, K., Philipp, A. M., et al. (2010). Control and inference in task switching - A review. Psychological Bulletin, 136(5), 849-874.

Kiss, M., \& Eimer, M. (2008). ERPs reveal subliminal processing of fearful faces. Psychophysiology, 45(2), 318-326. doi: $10.1111 / \mathrm{j} .1469-8986.2007 .00634 . x$

Klotz, W., \& Neumann, O. (1999). Motor activation without conscious discrimination in metacontrast masking. Journal of Experimental Psychology: Human Perception and Performance, 25(4), 976-992.

Lamy, D., Mudrik, L., \& Deouell, L. Y. (2008). Unconscious auditory information can prime visual word processing: A process-dissociation procedure study. Consciousness and Cognition, 17(3), 688-698. doi: https://doi.org/10.1016/j. concog.2007.11.001

Lang, P., \& Bradley, M. M. (2007). The International Affective Picture System (IAPS) in the study of emotion and attention. Handbook of emotion elicitation and assessment, 29.

Lundqvist, D., Flykt, A., \& Öhman, A. (1998). The Karolinska directed emotional faces (KDEF). CD ROM from Department of Clinical Neuroscience, Psychology section, Karolinska Institutet.

Marcel, A. J. (1983). Conscious and unconscious perception: Experiments on visual masking and word recognition. Cognitive Psychology, 15(2), 197-237.

Martens, U., Ansorge, U., \& Kiefer , M. (2011). Controlling the unconscious: Attentional task sets modulate subliminal semantic and visuo-motor processes differentially. Psychological Science, 22(2), 282-291.

Martens, U., \& Kiefer, M. (2009). Specifying attentional top-down influences on subsequent unconscious semantic processing. Advances in Cognitive Psychology, 5, 56-68.

Mayr, U., \& Keele, S. W. (2000). Changing internal constraints on action: The role of backward inhibition. Journal of Experimental Psychology: General, 129(1), 4-26.

Neumann, O. (1984). Automatic processing: A review of recent findings and a plea for an old theory. In W. Prinz \& A. F. Sanders (Eds.), Cognition and motor processes (pp. 255-293). Berlin: Springer.

Öhman, A., Flykt, A., \& Esteves, F. (2001). Emotion drives attention: Detecting the snake in the grass. Journal of Experimental Psychology: General, 130(3), 466-478.

Oldfield, R. (1971). The assessment and analysis of handedness: The Edinburgh Inventory. Neuropsychologia, 9, 97-113.

Palermo, R., \& Rhodes, G. (2007). Are you always on my mind? A review of how face perception and attention interact. [Review]. Neuropsychologia, 45(1), 75-92. doi: 10.1016/j.neuropsychologia.2006.04.025

Pessoa, L., \& Adolphs, R. (2010). Emotion processing and the amygdala: from a'low road'to'many roads' of evaluating biological significance. Nature reviews neuroscience, 11, 773-782. doi: https://doi.org/10.1038/nrn2920

Posner, M. I., \& Snyder, C. R. R. (1975). Attention and cognitive control. In R. L. Solso (Ed.), Information processing and cognition: The Loyola Symposium (pp. 55-85). Hillsdale: Lawrence Erlbaum Associates.

Rogers, R. D., \& Monsell, S. (1995). Costs of a predictible switch between simple cognitive tasks. Journal of Experimental Psychology: General, 124(2), 207-231.

Schmitz, M., \& Wentura, D. (2012). Evaluative priming of naming and semantic categorization responses revisited: A mutual facilitation explanation. Journal of Experimental Psychology: Learning, Memory, and Cognition, 38(4), 984-1000. doi: $10.1037 / \mathrm{a} 0026779$

Schmitz, M., Wentura, D., \& Brinkmann, T. A. (2014). Evaluative priming in a semantic flanker task: ERP evidence for a mutual facilitation explanation. Cognitive, Affective \& Behavioral Neuroscience, 14(1), 426-442. doi: 10.3758/s13415-013-0206-2

Schneider, W., \& Shiffrin, R. M. (1977). Controlled and automatic human information processing: I. Detection, search, and attention. Psychological Review, 84(1), 1-66.

Spruyt, A., De Houwer, J., \& Hermans, D. (2009). Modulation of automatic semantic priming by feature-specific attention allocation. Journal of Memory and Language, 61(1), 37-54.

Tanaka, J. W., Kiefer, M., \& Bukach, C. M. (2004). A holistic account of the own-race effect in face recognition: Evidence from a cross-cultural study. Cognition, 93(1), B1-9. doi: 10.1016/j.cognition.2003.09.011

Vorberg, D., Mattler, U., Heinecke, A., Schmidt, T., \& Schwarzbach, J. (2003). Different time courses for visual perception and action priming. Proceedings of the National Academy of Sciences of the United States of America, 100(10), 6275-6280.

Wentura, D., \& Rothermund, K. (2003). The "meddling-in" of affective information: A general model of automatic evaluation effects. In J. Musch \& K. C. Klauer (Eds.), The psychology of evaluation: Affective processes in cognition and emotion (pp. 51-86): Erlbaum.

Zovko, M., \& Kiefer, M. (2013). Do different perceptual task sets modulate electrophysiological correlates of masked visuomotor priming? Attention to shape and color put to the test. Psychophysiology, 50(2), 149-157. doi: 10.1111/j.1469. 8986.2012.01492.x 